\title{
An Innovative Approach about the Process Knowledge Representation in the Processes of Large Cluster Projects Management
}

\author{
Yong Liu, Qiong Chen
}

School of Business Administration, South China University of Technology, Guangzhou, China.

E-mail: liuyong@scut.edu.cn, cqjoanchen@163.com

Received July $28^{\text {th }}, 2010$; revised September $7^{\text {th }}, 2010$; accepted October $23^{\text {rd }}, 2010$.

\begin{abstract}
The features of the large Cluster projects' management processes, complexity, "one-off" and "irreversibility", make the projects' process knowledge hard to be expressed, analyzed and obtained accurately. It is also not convenient to be accumulated and disseminated, and can't be learned and used by managers. However, such knowledge is the crystallization of human wisdom. It has an important role in promoting efficiency of projects management and increasing accumulation of social knowledge. A better way is needed to find for its representation and utilization. From the perspective of cluster project management department, this article takes a cluster project's construction as an example, and proposes a suitable method for the process knowledge representation in large cluster projects' management processes, combined with the Topic Maps and MFFC-II \& MLD- II.
\end{abstract}

Keywords: Cluster Projects, Process Knowledge, Knowledge Representation

\section{Introduction}

With rapid development of economic and social progress, large-scale trend of projects becomes more and more obvious. A number of complexity cluster projects covering many areas in large scale, distributed information and Cross Specialized fields are emerging, such as Olympic project, Expo project, and Asian Games projects. Mainly composed of a number of large projects, these luster projects are generally carried out under cluster project management department. They need huge investment and have huge influence in the world [1]. Meanwhile, large cluster projects with large scale and complexity processes usually aim at particular purposes. With "one-off" and "irreversibility" feature, they are calling for "one-off things done" [2]. It is a high demand for projects management, and also a challenge to cluster project management department's management and policy-making.

Processes of large cluster projects' management by government have rigorous characteristics and contain a lot of knowledge, which is hard to be accumulated, organized and represented because of the characteristics of

Supported by "the Fundamental Research Funds for the Central Universities, SCUT”, project number: 2009ZM0115. the large cluster projects, such as complexity, one-time and irreversibility, but has a great guide and good reference for other similar projects' management, and has great significance in the improvement of the project results, the project efficiency, even the accumulation of the whole society's knowledge. If the process knowledge in large cluster projects' management can't obtain better representation, acquisition, sharing and dissemination, it will be very difficult to guide the projects' implementation. Problems in the projects can't be solved intermediately; time of the projects' construction can't be managed efficiently; project funding can't be used sensibly and the whole projects can't be completed in accordance with the intended targets. How terrible the results are!

We need to create a convenient and practical process knowledge representation method to express process knowledge which are comprehensively, clearly and compactly contained in cluster projects' management, if we want to access, share and transfer it. That is the problem which needs to be studied and solved urgently.

\section{Process Knowledge and Process Knowledge Representation}

Process knowledge includes procedures, relevant regula- 
tions and knacks to achieve specific targets of the task. It's a kind of procedural, methodological and combinational knowledge mostly obtained from the practice of "doing". The actives and the relationships between are the cores of it [3].

Currently, major representation methods of process knowledge are graphic representation, ontology representation and so on. Graphic representation, such as process knowledge map [4], MFFC (multi-factor flow chart), MLD (multi-level text), hierarchical task network [5], data flow diagram [6], super-graph model [7], and the event process chain [8], is an illustrated knowledge representation method which is easy, clear and concise. Ontology representation is the dominant representation of concept model. On surface, there are not many differences between "ontology" and data dictionary. They are both collection of many terms. But ontology is more rigorous and precise than data dictionary in content. The grammar and the axioms of ontology should be elaborated in precise and formal language, syntax and clear defined semantics, such as the PKOR [9] program based on KIF, IDEF5 [10] and system modeling [11], the XML language description [12] and so on. However, these approaches were often used in enterprises for the representation of business process knowledge but can't meet with the requirements of the large cluster projects because of their characteristics of large scale, high technical difficulty, long construction period, and complexity environment [13].

\section{Characteristics of Process Knowledge in the Processes of Large Cluster Projects Management}

The processes of large cluster projects management are complex and changeable. So process knowledge contained in it is very complicated and diverse. To represent process knowledge well, we should have a more in-depth knowledge and understanding of its characteristics.

In the processes of large cluster projects management, the order of management processes is very clear for individual project. Only after the accomplishment of the first step, can the second step be put into practice. So, strong sequential representation methods of process knowledge should be better for its representation. However, if standing in the management level of large cluster projects, we can find that, there are no very strict order relations among the components. To achieve the objective as soon as possible, with sufficient funding, personnel, materials and so on, many projects are parallel implementation, and are not appropriate to use strong sequential process knowledge representation methods. Evidently, a single process knowledge representation method can't be a good way to represent the process knowledge in the processes of large cluster projects management. A more appropriate approach is to combine a strong sequential method with a non-strong one, integrate their advantages and form a new representation method.

Topic map is a knowledge representation model similar to semantic network. Combining the advantage of traditional indexing, library science, artificial intelligence and any other fields, it can organize the knowledge efficiently to probe, infer and solve questions caused by large number of disordered information [14]. It's a suitable method for the representation of non-strong sequential process knowledge in the processes of large luster projects management.

MFFC- II \& MLD- II (the improved multi-factor flow chart and the improved multi-level text), which were proposed by Dr. Guo Weisen, is a representation method based on MFFC \& MLD. MFFC-II expresses main process steps in process knowledge, while MLD- II expresses activities and relations in detail. Descriptions of knowledge input and output are added to MFFC-II, so that the document knowledge can correspond to the management processes, and be managed well. MFFC - II \& MLD- II is a suitable method to express strong sequential process knowledge in the processes of large cluster projects management [15].

Therefore, this article try to combine topic maps with MFFC- II \& MLD- II, and to create a new and suitable method for better representation of process knowledge in the processes of large cluster projects management. That will lay the foundation for process knowledge's sharing and transferring.

\section{Topic Map and MFFC- II \& MLD- II}

\subsection{Topic Map [16]}

The topic map can be used to describe and manage the complex information word. It consists of three basic elements: Topic (T), Occurrence (O) and Association (A). Topic can represent any meaningful projects, such as name, organization, concept, location, etc. It is a basic unit in topic map to express knowledge. Any object which can lead the user to discuss should be included in it. Based on application requirements, topics can be divided into different categories, named topic types. A topic type is also a topic. The relationship between a topic and a topic type is equivalent to that between a class and its instance. A topic can link to one or more related information resources. Description of these resources is called the occurrence of the topic, and usually exists in the form of documents, Web pages and so on. Association means the relationship between two or more 
topics. Like topics, association can also be divided into different categories, called association types. For example, "among the...", "participate in ..." and so on. Association types are also topics, and are defined in the form of topics. Among topics, all types of relationship which are organized and linked by associations will form a knowledge network. Just through $\mathrm{T}, \mathrm{O}$, A these three elements, topic maps organize information resources, establish indexes, and construct semantic network by describing the relationships among topics. So they support the accurate location of interested knowledge points and related information resources.

\subsection{MFFC- II \& MLD- II [15]}

MFFC- II \& MLD- II are proposed on the base of MFFC $\&$ MLD. The basic syntax elements of MFFC contain the based map of body- time, activities rectangular box, relationship arrows (different arrow types mean different relationships, including four relationships: order, and, or, and xor) and time line. Based on MFFC, MFFC- II adds knowledge input and output, and closely combines flow technology with knowledge which is needed or obtained or produced in the processes of projects management, as is shown in Figure 1 and Figure 2.

MLD- II is the detail description of activities and relationships in the MFFC- II . It adds descriptions of knowledge input and output based on MLD, as is shown in Figure 3 and Figure 4.

\section{The Study of Representation Method}

According to the characteristics of large cluster projects' management processes, combined with topic map and MFFC- II \& MLD- II, the new representation method of process knowledge in the processes of large cluster projects management is that:

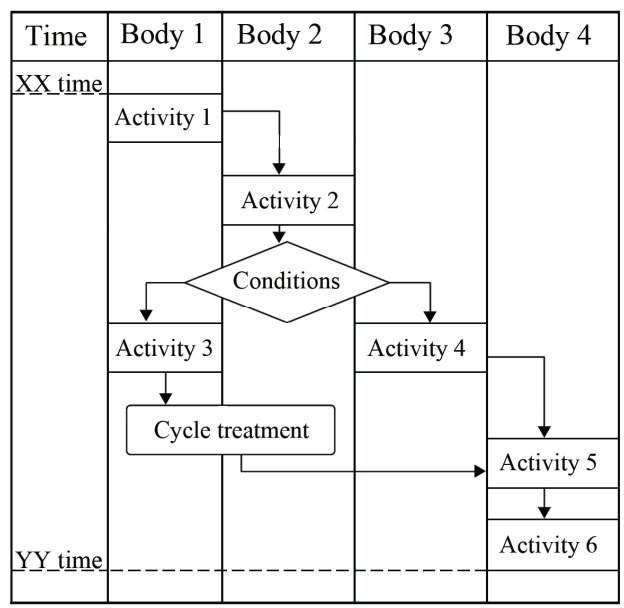

Figure 1. The schematic diagram of MFFC.

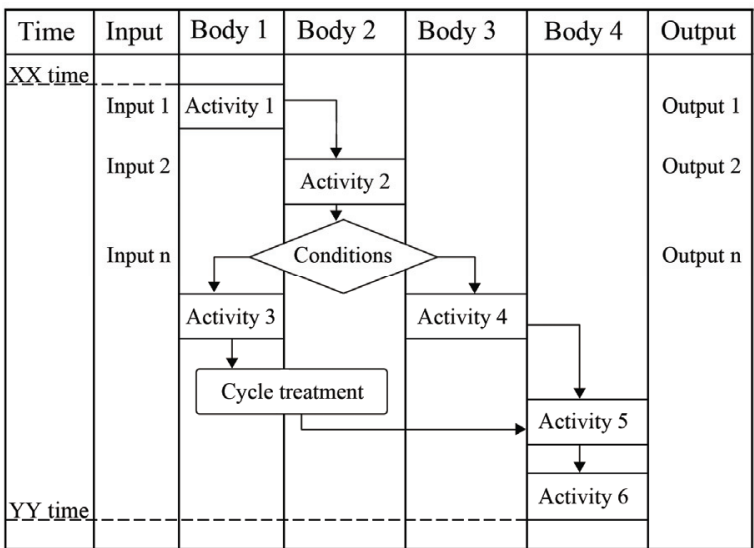

Figure 2. The schematic diagram of MFFC-II .

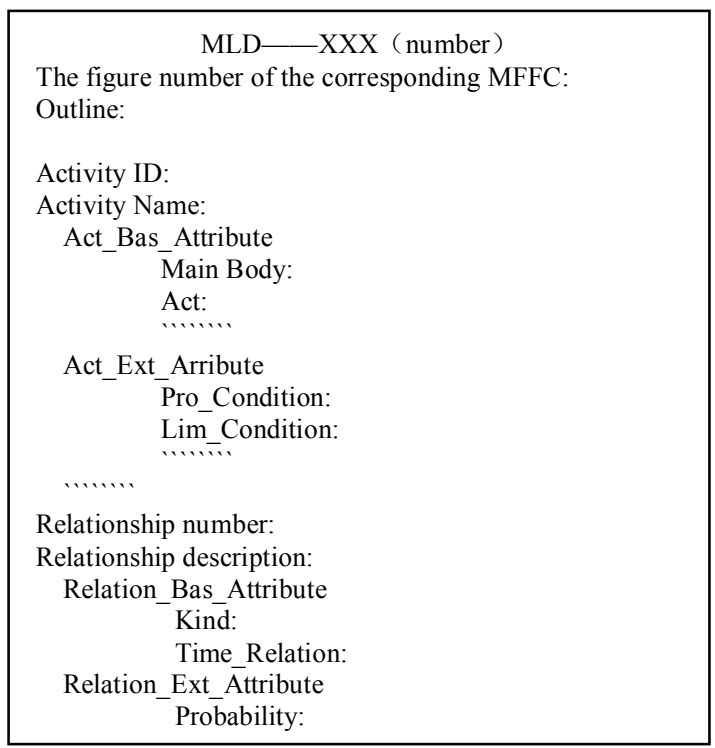

Figure 3. The schematic diagram of MLD.

Step one: Take each component project as a topic and divide the large cluster projects

Large cluster projects are composed of a number of large construction projects which have no strict order relations but sharing, occupation, mobility relations among funds, staff, and materials. It is suited for the representation method of topic map and can be classified into various topics. If the number of components is large, we can firstly classify topic types, and then topics.

Step two: Sort out and number the documents produced in the management processes

There are many files and documents in cluster project management department's management processes. They are important parts of knowledge input and output. Numbered by different topics, operational links or types, they will be more conducive to be found and used. This 


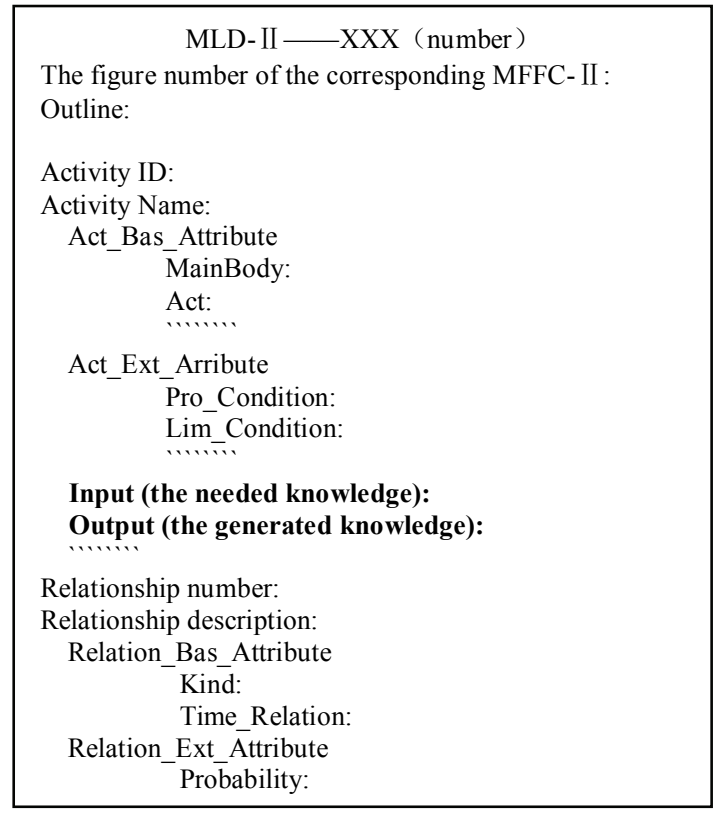

Figure 4. The schematic diagram of MLD- II.

step is the base of MFFC- II \& MLD- II 's drawing. Document numbering can be carried out during the projects implementation processes. Timely classification will help the acquisition of processes knowledge.

Step three: draw MFFC- II charts under each topic

As the whole implementation processes of one single large project have strict operational processes, its management process knowledge can be represented clearly and coherently by MFFC- II charts.

Step four: draw MLD- II charts for activities and relations in the MFFC- II charts

Drawing MLD- II charts is to obtain detail descriptions of activities and relations in MFFC- II charts. It can describe the projects' process knowledge well. In the meantime, a MLD- II chart is also a bridge between topics and occasions. It links topics and projects' management knowledge (the management files), and forms an organic whole to represent the projects' process knowledge completely.

Step five: systematic integration

All the steps above are not isolated, but integrated together systematically. They are interrelated and complementary. Systematic integration can make the form of representation clearer, content more complete, and process more convenient.

Systematic integration can be divided into four levels: the top level is the topic layer of cluster projects. The second level is the layer of MFFC- II chart corresponding to each topic. The third level is the MLD- II chart layer. The fourth level is the occasion layer which is the set of the documents in the processes of management. Each level is closely linked, as is shown in Figure 5.

\section{Case Study}

This representation method of process knowledge in large cluster project management has already applied in some management processes of cluster project's construction. For example, a project is composed of seven major parts including athlete village, technical official village, mass media village, sports venues, press center, administrative service area and Theme park. It is presided and planned by the cluster project management department and regarded as a typical large cluster project. Because, there are many projects involved, we can classify the topic types by the projects' types at first. Then, classify topics, as shown in Figure 6.

Taking the building of gymnastics stadium as an example, we can draw a MFFC- II chart of the management processes after numbering the documents generated in the management processes systematically. The MFFCII chart in creation stage of gymnastics stadium construction is as shown in Figure 7.

Among them, taking the activity of feasibility analysis as an example, the corresponding MLD-II chart is as shown in Figure 8.

Finally, we combine project topics, MFFC- II charts, MLD- II charts, and documents (events) generated in the management processes together, and they form a system integration map of the Cluster projects as shown in Figure 9. Thereby, it can clearly express the process knowledge in the processes of Cluster projects management.

\section{Conclusions}

Because of different characteristics of large cluster projects, there are many difficult issues in representation, acquisition, analysis and sharing of large cluster project's

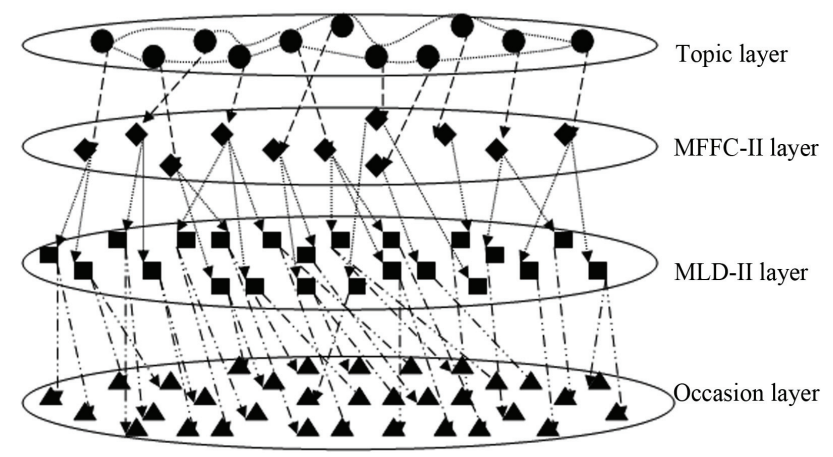

Figure 5. The integration schematic diagram of process knowledge's representation method in the large cluster project management. 


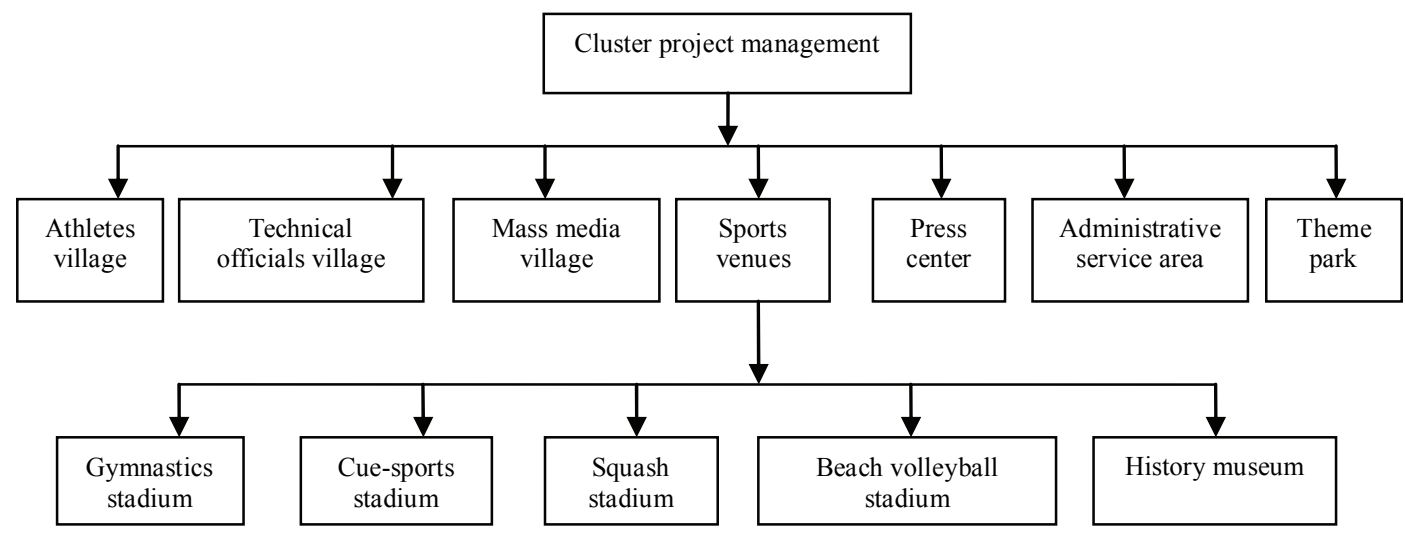

Figure 6. The topics' partition chart of cluster project management.

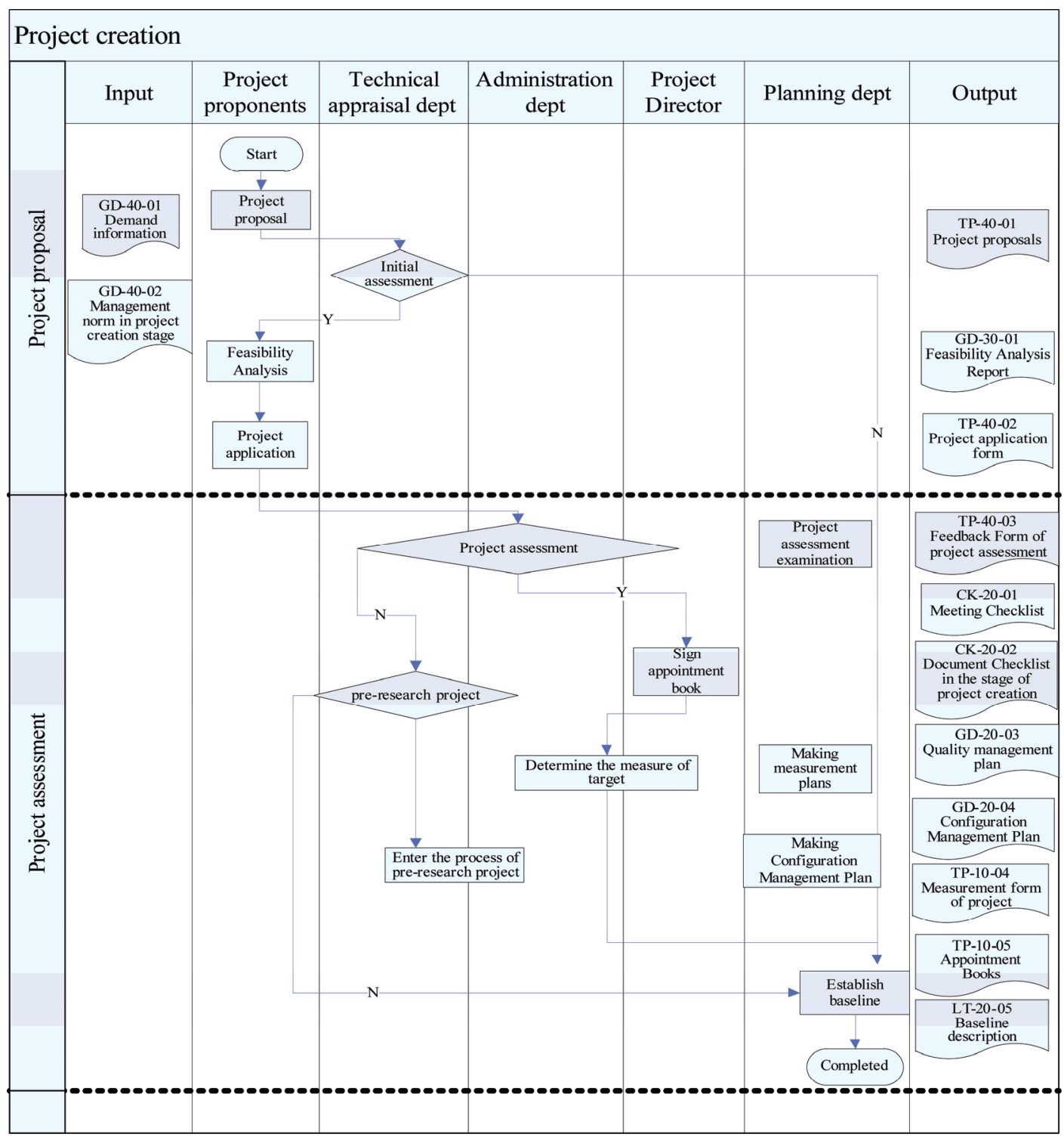

Figure 7. MFFC-II chart in the creation stage of gymnastics stadium construction. 
MLD- II — the activity of feasibility Analysis (01-01-03)

The corresponding figure No. of MFFC- II : 01-01

Description: the MFFC- II chart in the stage of project creation of

gymnastics stadium's construction

Activity number: 01-01-03

Activity name: Feasibility analysis

Basic attributes

Body: Li $\times \times$, Wang $\times \times$, Zhao $\times \times$

Action: To making the feasibility analysis of gymnastics stadium's construction

Time: project application stage

Extended attributes

Prerequisite: Gymnastics stadium's construction had passed the initial assessment

Constraints: None

Participate: Li $\times \times$, Wang $\times \times$, Zhao $\times \times$

Input(knowledge needed): GD-40-01 demand information

Output (knowledge generated): GD-30-01 feasibility analysis report

Figure 8. The corresponding MLD- II chart of feasibility analysis activity.

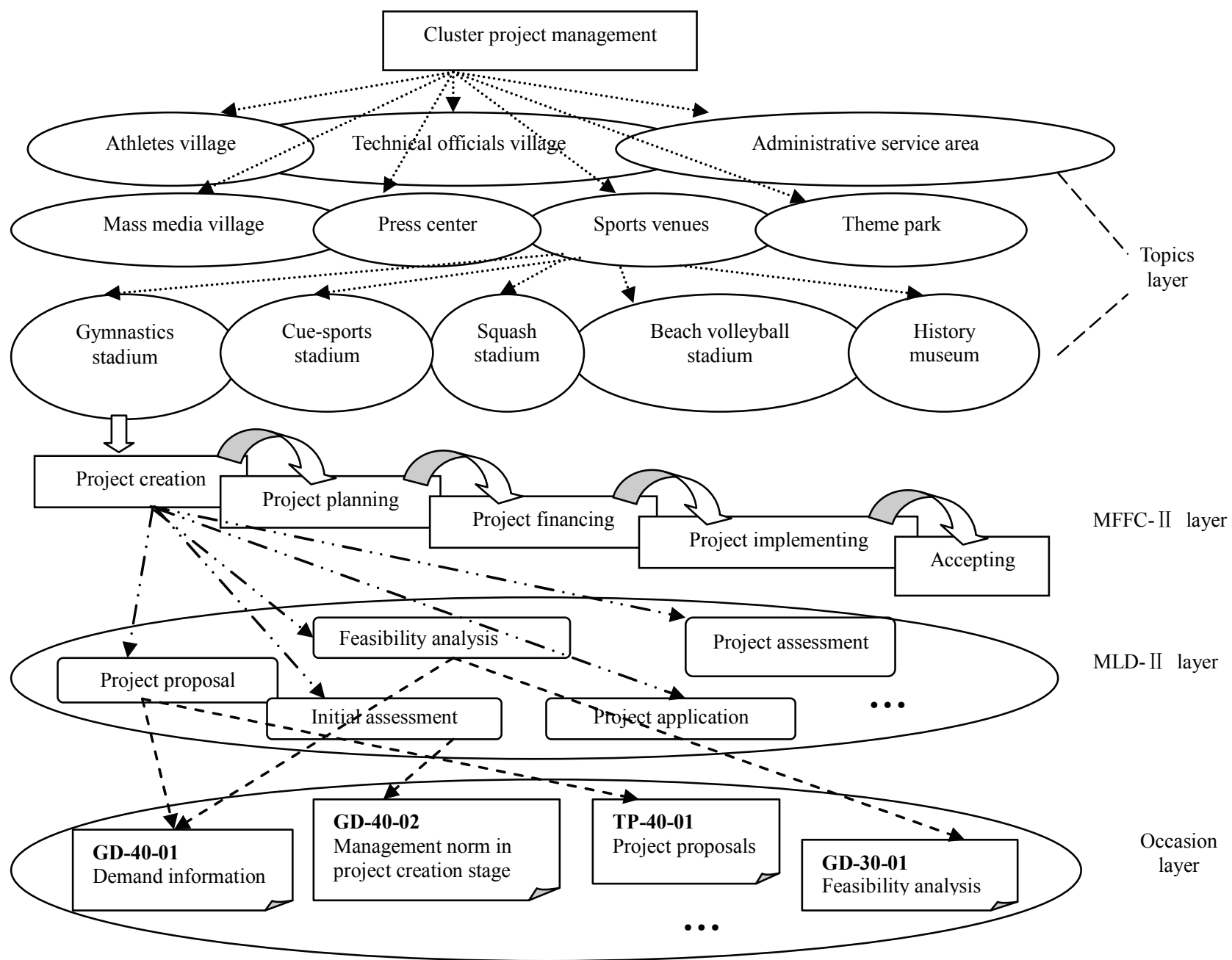

Figure 9. The system integration map of the cluster projects' process knowledge representation. 
management process knowledge. However, there is a large number of process knowledge in the processes of large cluster projects management which can be learned and be re-used. It is necessary to express, analyze, access, Share and transfer process knowledge in the management processes. Combined with the Topic Maps and MFFC-II \& MLD-II, this article forms a new method of process knowledge representation and gives specific implementation steps. It lays the foundation for analysis, acquisition, sharing and transferring of the knowledge, and provides relevant reference for the management of similar projects. This method is simple, clear, easy to understand, and has wide application prospect.

\section{REFERENCES}

[1] L. Z. She, "Research on Quality Supervision of Clusters of Engineering Projects Based on Science of Complexity," Journal of Chongqing Jianzhu University, Vol. 28, No. 3, June 2006, pp. 107-109.

[2] S. Jeong Wook, "Eddy M. Understanding Collaborative Working Processes of Temporary Project Teams in Large-Scale Construction Projects," Construction Research Congress - Building a Sustainable Future, April 2009.

[3] W. S. Guo and Y. Z. Dang, "A Process Knowledge Acquiring Method Based on Business Process in Corporation," Chinese Journal of Management Science, December 2003, pp. 53-58.

[4] J. Liang and Z. H. Jiang, "The Research and Application of Process Knowledge Map Constructing Method," Journal of the Chinese Institute of Industrial Engineers, January 2007, pp. 30-41.

[5] K. Erol, J. Hendler and D. S. Nau, "Semantics for Hierarchical Task Network Planning," Technical Report, Computer Science Depatment, ISR, UMIACS, University of Maryland, College Park, March 1994.

[6] A. P. Barros, A. H. M. Hofstede, H. A. Proper and P. N.
Creasy, "Business Suitability Principles for Workflow Modelling," Technical Report 380, Department of Computer Science, University of Queensland, Brisbane, August 1996.

[7] N. F. Cui and R. Q. Chen, "Study on Lead-Time of Business Process," Journal of Industrial Engineering and Engineering Management, Vol. 15, No. 2, 2001, pp. 62-64.

[8] J. Z. Li and L. Y. Chen, "Extended Event-Process Chain (EEPC) and its Application in BPR," System Engineering, Vol. 18, No. 1, pp. 42-48.

[9] Y. Q. Gong, M. Lu and W. Gang, "Research on Process Knowledge Management Based on Ontology," International Conference on Wireless Communications, Networking and Mobile Computing, September 2007, pp. 21-25.

[10] P. C. Benjamin et al., "IDEF5 Report, KBSI," 1994.

[11] J. van Beveren. "A Model of Knowledge Acquisition that Refocuses Knowledge Management," Journal of Knowledge Management, June 2002, pp. 18-22.

[12] Y. Mao, Q. Chen and R. Z. Yu, "XML: A Data Format Description Language Which Will be Widely Used," Application Research of Computers, September 1999, pp. 4-5.

[13] J. L. Zhang, L. Sun and J. Y. Chi, "An Approach to Risk Analysis of Project Bidding Based on Rough Sets," Journal of Huazhong University of Science and Technology, Vol. 30, No. 9, September 2002, pp. 45-47.

[14] L. Zhang and Y. Cheng, "Overview of Research on Topic Maps," Information Science, Vol. 27, No. 2, February 2009, pp. 305-309.

[15] Y. Liu, Z. T. Wang and Q. Chen, "The Research on the Method of Process Knowledge Representation and Acquisition in the Process of Project Management," International Conference on Management and Service Science, September 2009.

[16] X. F. Wu, Z. Liang, L. Zhang and Q. L. Ding, "Knowledge Warehouse Based on XML Topic Maps," Engineering Journal of Wuhan University, Vol. 39, No. 1, February 2006, pp. 121-125. 